\title{
A novel Whole Air Sample Profiler (WASP) for the quantification of volatile organic compounds in the boundary layer
}

\author{
J. E. Mak ${ }^{1}$, L. Su ${ }^{1}$, A. Guenther ${ }^{2, *}$, and T. Karl $^{2, * *}$ \\ ${ }^{1}$ School of Marine and Atmospheric Sciences, Stony Brook University, Stony Brook, New York, USA \\ ${ }^{2}$ National Center for Atmospheric Research, Boulder, Colorado, USA \\ * now at: Pacific Northwest National Laboratory, Richland, Washington, USA \\ ** now at: Department of Meteorology and Geophysics, Karl Franzens University, Innsbruck, Austria
}

Correspondence to: J. E. Mak (john.mak@stonybrook.edu)

Received: 16 April 2013 - Published in Atmos. Meas. Tech. Discuss.: 6 May 2013

Revised: 27 August 2013 - Accepted: 5 September 2013 - Published: 16 October 2013

\begin{abstract}
The emission and fate of reactive volatile organic compounds (VOCs) is of inherent interest to those studying chemical biosphere-atmosphere interactions. In-canopy VOC observations are obtainable using tower-based samplers, but the lack of suitable sampling systems for the full boundary layer has limited the availability of data characterizing the vertical structure of such gases above the canopy height and still in the boundary layer. This is an important region where many reactive VOCs are oxidized or otherwise removed. Here we describe an airborne sampling system designed to collect a vertical profile of air into a $3 / 8 \mathrm{in}$. OD (outer diameter) tube $150 \mathrm{~m}$ in length. The inlet ram air pressure is used to flow sampled air through the tube, which results in a varying flow rate based on aircraft speed and altitude. Since aircraft velocity decreases during ascent, it is necessary to account for the variable flow rate into the tube. This is accomplished using a reference gas that is pulsed into the air stream so that the precise altitude of the collected air can be reconstructed post-collection. The pulsed injections are also used to determine any significant effect from diffusion/mixing within the sampling tube, either during collection or subsequent extraction for gas analysis. This system has been successfully deployed, and we show some measured vertical profiles of isoprene and its oxidation products methacrolein and methyl vinyl ketone from a mixed canopy near Columbia, Missouri.
\end{abstract}

\section{Introduction}

Volatile organic compounds (VOCs) are emitted from almost all plant species (Fuentes et al., 2000; Kesselmeier and Staudt, 1999; Laothawornkitkul et al., 2009). These biogenic emissions can dominate regional atmospheric chemistry (Atkinson and Arey, 2003; Guenther et al., 2000). As a result, numerous field studies designed to quantify biogenic VOC (BVOC) emissions have been executed (Karl et al., 2007; Bouvier-Brown et al., 2009; Kim et al., 2010; DiGangi et al., 2011), and future campaigns are imminent.

One of the goals in these field campaigns is to determine the flux of VOCs from the forest canopy. Typically a tower is erected at a forest site and sampling lines are installed at various elevations. Measurements from multiple heights along the tower can characterize the structure of the vertical profile of VOCs. Direct flux measurements can also be used, if one has a fast response trace gas measurement system. In this case an eddy covariance (EC) sampling line is used for realtime measurements of VOC concentrations that are then correlated with vertical wind speed, $w$, as measured by a sonic anemometer located on the same tower (Müller et al., 2010; DiGangi et al., 2011). From this information, along with measurements of other atmospheric constituents, researchers can determine emissions rates and describe chemistry and transport within the forest canopy.

Ground-based measurements are very useful for quantifying chemical and physical processes that are occurring within the forest canopy (Karl et al., 2007; Kim et al., 2010). There are few measurements, however, of VOC concentrations 


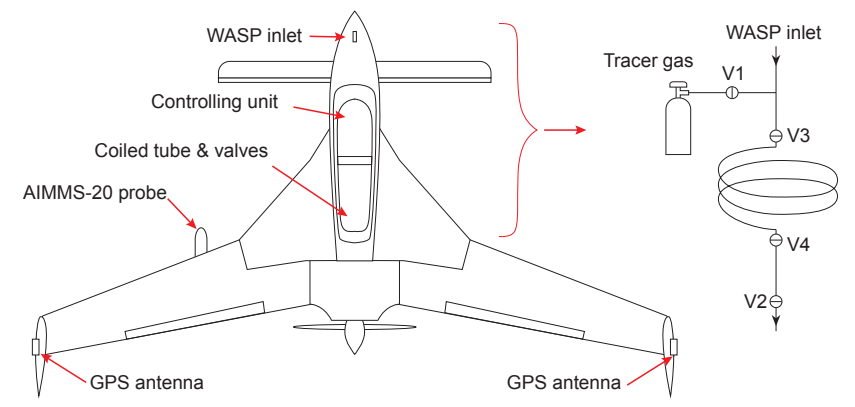

Fig. 1. Schematic drawing of the long-EZ aircraft and WASP. V1 and V2 are solenoid valves, V3 and V4 are ball valves.

above the forest canopy. As can be imagined, measurements above the tower height within a forest are difficult to make. To date the most common approach to obtain vertical profiles of VOCs has been from a balloon platform (Greenberg et al., 1999). Balloons fitted with three to four small air samplers (pumps) are flown to a height of perhaps a few hundred meters above ground and suspended for a certain length of time, usually 20 minutes to one hour, after which collected air samples are retrieved and analyzed. While useful, balloons have several limitations: they can only be flown in clearings, and not within a forest canopy; a small number of samplers means that the vertical resolution is poor; maximum sampling altitude above ground is relatively low, and is usually not sufficient to reach the top of the mixed boundary layer; and the height of a balloon can vary significantly during sample collection, because of changes in wind strength.

Another method for determining trace gas fluxes is to use an aircraft outfitted with a fast response analyzer and a wind measurement system. This approach has been used successfully to determine the fluxes of VOCs such as isoprene and is the most direct technique for measuring trace gas fluxes. However, only two studies have used this technique: the 2006 MIRAGE study (Karl et al., 2007), and the 2011 CABERNET study (Karl et al., 2013). Other direct airborne VOCs measurements were also carried out by using PTR-MS (de Gouw and Warneke, 2007; Warneke et al., 2010). The reason for this is simply the cost; one needs a dedicated analytical instrument, and more importantly, one needs an aircraft large enough and sophisticated enough to make the wind measurements. The aircraft cost is therefore often prohibitive, except for a few large field campaigns. Thus there is a need for a more affordable, simpler method to obtain the information on VOC profiles above canopy height.

Here we describe an air sampling system that can be deployed on a small single engine aircraft and used to collect vertical profiles of air. We also show some initial results for vertical profiles of isoprene and its oxidation products methyl vinyl ketone (MVK) and methacrolein (MACR) over a forest canopy.

\section{Experimental methods}

\subsection{Description of WASP}

The aircraft used to collect the profiles presented here is a long-EZ with a single rear engine (Fig. 1). The cruise speed (true air speed) of the aircraft is $30-100 \mathrm{~m} \mathrm{~s}^{-1}$. The air sampling inlet ( $1 / 2$ in. OD (outer diameter) stainless steel tubing) is installed at the front of the aircraft. The inlet is connected to a $1 / 2$ in. ID (inner diameter) perfluoroalkoxy (PFA) tube and connected to the coiled tube located in the rear of the aircraft cockpit (Fig. 1). The WASP (Whole Air Sample Profiler) was used to collect air in the boundary layer above a forest canopy, the air samples were analyzed offline by using a PTR-TOF-MS (proton-transfer-reaction time-of-flight mass spectrometer) operated in the laboratory (see Sect. 2.3).

The working principle behind the WASP is straightforward. Air from outside of the aircraft flows into a long $(150 \mathrm{~m})$ tube during flight, using the ram air pressure resulting from the aircraft's speed. An automated ball valve, placed at the back end of the tube and controlled by the pilot, is opened a few moments before sampling, to flush the tubing. Sample collection proceeds when the aircraft begins to climb, and stops when the valve is closed by the pilot, which is at a time calculated to be the sample fill time, or about 2 min, depending on aircraft speed and altitude. The aircraft used here has an average climb rate of about $500 \mathrm{~m} \mathrm{~min}^{-1}$, so a 2 min collection time results in a vertical profile of about $1000 \mathrm{~m}$. The aircraft speed and ascent rate is greater at the beginning of the climb, as it is usually preferable to get the tallest vertical profile possible. However, the changing aircraft speed results in a reduced ram pressure and thus a changing velocity through the sample tube. We wish to know precisely the altitude at which the sample is collected throughout the length of the tube, so we use a chemical tracer to "mark" the air sample at discrete intervals. The chemical tracer (in this case, propene, $1 \mathrm{ppmv}$ ) is pulsed into the airstream at fixed intervals; we found that a $0.2 \mathrm{~s}$ pulse every 5-10 s resulted in well resolved peaks, but expect this will be dependent upon the specifics of the air sample flow rate and chemical tracer. The tracer pulses are analyzed, in this case by PTR-TOF-MS (along with the rest of the air sample) postcollection and then reconstructed and correlated with the fast GPS altitude system installed in the aircraft. This approach allows the calculation of the altitude of the sample air within the tube to within a few meters. The tracer pulses are also used to characterize the degree of mixing and/or smearing due to either diffusion or Poiseuille flow during extraction of air from the tube for analysis.

The $150 \mathrm{~m}$ tube used here is $3 / 8 \mathrm{in}$. OD 304 stainless steel with $0.02 \mathrm{in}$. wall thickness, in sections of $30 \mathrm{~m}$. The entire tube is coiled to a diameter of about $24 \mathrm{in}$. Ball valves are fitted onto each end, and on the back of the tubing, a solenoid valve (ASCO Valve, Inc.) is installed for automated closing during flight. The stainless steel was cleaned with reagent 
grade ethanol and rinsed multiple times with DI (deionized) water, then heated for $24 \mathrm{~h}$ to $60^{\circ} \mathrm{C}$ under a low flow of zero air generated by a catalytic converter ( $\mathrm{Pt}$ wool heated to $350^{\circ} \mathrm{C}$ ).

The aircraft-based sampling system consists of two separate components: (1) the air sampling component called WASP, presented here; and (2) the onboard meteorological data monitoring system (the Aircraft-Integrated Meteorological Measurement System (AIMMS-20), Aventech Research Inc.). The latter provides GPS positioning and realtime winds in three dimensions at a frequency of $20 \mathrm{~Hz}$ and has been evaluated previously (Beswick et al., 2008).

The WASP has three sampling modes: inactive, flushing and sampling. In inactive mode, typically, before the aircraft reaches the desired sampling altitude, the WASP is isolated. Both valve V1 and V2 are closed while V3 and V4 are open (Fig. 1), and no ambient air is drawn into the coiled tube. In flushing mode, typically starting a few minutes before the aircraft reaches the sampling location, valve $\mathrm{V} 2$ is opened by the pilot, and ambient air is flushed into the coiled tube. In sampling mode, once the aircraft reaches the sampling location, the pulsed injection system is initiated (again by the pilot). At this point, a tracer gas is injected into the air stream at discrete intervals, by the automated cycling of valve V1. The ambient meteorological data (e.g., temperature, relative humidity, wind direction and speed) are monitored by the onboard AIMMS-20 system. The latitude, longitude and altitude are also logged simultaneously by using the integrated GPS antenna (Fig. 1). The controlling and data logging of WASP and AIMMS-20 are accomplished by using an onboard PC running LabVIEW. After one sampling flight is finished, the WASP is switched back to inactive mode with V1 and V2 closed. After the aircraft lands, the coiled tube containing the air sample is sealed by manually closing ball valves V3 and V4 and transported to the lab for analysis (by using PTR-TOF-MS; see Sect. 2.3 for analytical description).

\subsection{Characterization of WASP}

\subsubsection{Turbulent versus laminar flow during sample collection}

It is ideal to collect an air sample under turbulent flow conditions. This minimizes the effect of molecular diffusion and Poiseuille flow, and results in a well mixed sample. Thus, the flow rate within the tube needs to be high enough to induce turbulence. Thus a high flow rate is needed to ensure turbulent flow, but it is also desirable to collect the tallest/longest vertical profile possible; and with increasing tube length, flow rate decreases at constant inlet pressure. Thus ideally one would want a large diameter, long tube. For a straight tube, turbulent flow is typically characterized by a Reynolds number $(R e)$ greater than about 4000 ; for a $3 / 8$ in. OD tube this corresponds to a flow rate of about $4-5 \mathrm{~m} \mathrm{~s}^{-1}$. For a $150 \mathrm{~m}$ length tube, this means a collection time of about
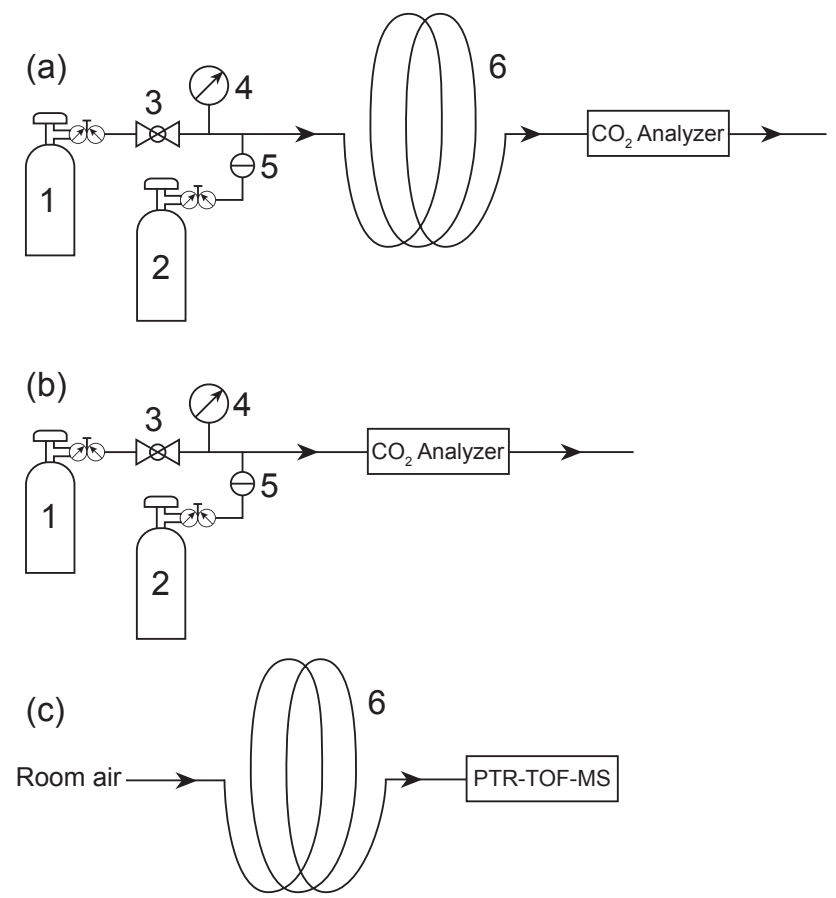

Fig. 2. The schematic drawings of the diffusion test system. 1: $\mathrm{N}_{2}$; 2: $10 \% \mathrm{v} / \mathrm{v} \mathrm{CO}_{2}$ or $1 \mathrm{ppmv}$ propene gas; 3 : ball valve; 4 : pressure gauge; 5: two-way valve; $6: 3 / 8 \mathrm{in}$. OD coiled tube ( $150 \mathrm{~m}$ length). (a) $\mathrm{CO}_{2}$-spiked $\mathrm{N}_{2}$ gas flows through the WASP tube into the $\mathrm{CO}_{2}$ analyzer; (b) $\mathrm{CO}_{2}$ spiked $\mathrm{N}_{2}$ gas goes directly into the $\mathrm{CO}_{2}$ analyzer; (c) after the propene-spiked $\mathrm{N}_{2}$ gas fills the WASP tube, the gas is analyzed with PTR-TOF-MS under a lower withdrawal flow rate.

$30 \mathrm{~s}$, which would result in a vertical profile of no more than $400 \mathrm{~m}$. This is much less than a typical summertime boundary layer height, and ideally, one would want a vertical profile from canopy height to the top of the mixed layer. The capability to obtain a vertical profile extending $1000 \mathrm{~m}$ above the canopy would be preferable.

Excessive weight and size makes the use of longer tube lengths impractical and so, despite its limitations, the $150 \mathrm{~m}$ length ( $3 / 8$ in. OD) tubing was deemed acceptable for our purposes. $R e$ of 4000 may be a very conservative minimum for our application, for two reasons: transient flow, which is nonlaminar, occurs in straight tube flow at $R e$ as low as 2300; and our flow is through a tightly wound tube, not a straight tube, thus the airflow is subject to a constant acceleration. Thus we assume that turbulent flow for the WASP will be at a significantly lower flow rate than that calculated at $R e=4000$.

Laboratory studies were conducted in order to determine whether air will be collected under a sufficiently turbulent flow regime during realistic aircraft sampling conditions. First, the ram pressure at the sample inlet of the aircraft was measured during flight at cruising speed. The observed ram pressure was measured to be about 110 mbar (near sea level), 


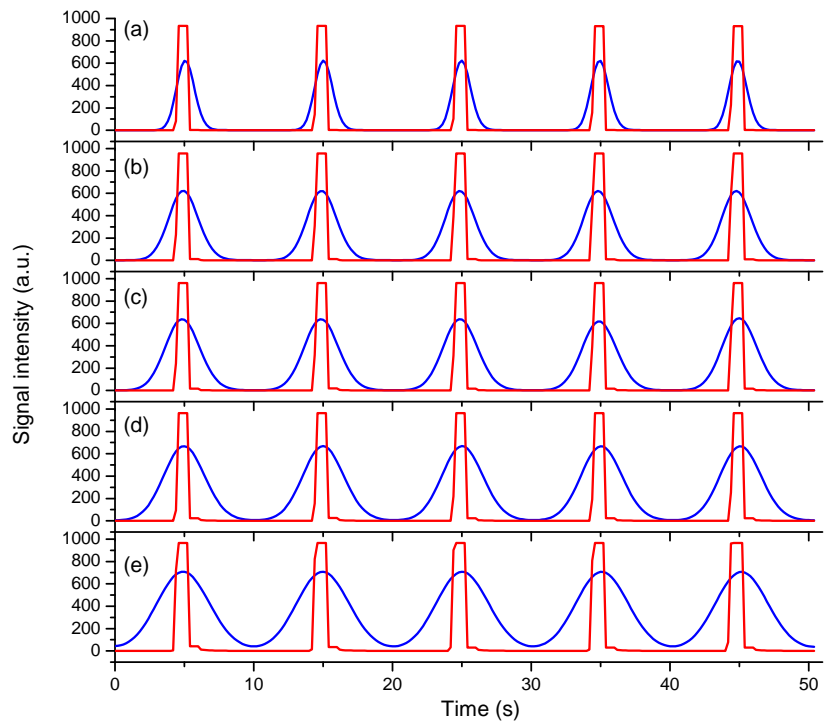

Fig. 3. Comparison of $\mathrm{CO}_{2}$ signals between pumping $\mathrm{CO}_{2}$-spiked $\mathrm{N}_{2}$ gas through the WASP tube (blue line) and pumping $\mathrm{CO}_{2}$-spiked $\mathrm{N}_{2}$ gas directly into the LI-COR $\mathrm{CO}_{2}$ analyzer (red line) under flow rates of: (a) $14 \mathrm{~L} \mathrm{~min}^{-1}$, (b) $7.7 \mathrm{~L} \mathrm{~min}^{-1}$, (c) $6.6 \mathrm{~L} \mathrm{~min}^{-1}$, (d) $5.4 \mathrm{~L} \mathrm{~min}^{-1}$, and (e) $3.9 \mathrm{~L} \mathrm{~min}^{-1}$, which correspond to a ram air pressure of $110,50,40,30$, and 20 mbar in front of the WASP tube, respectively.

which corresponds to a flow rate of 14 liters per minute $\left(\mathrm{L} \mathrm{min}^{-1}\right)$ through the tubing. Then, in the laboratory, $\mathrm{CO}_{2}$ in air $\left(10 \% \mathrm{v} / \mathrm{v} \mathrm{CO}_{2}\right.$ gas) was pulse injected into the entire length of tube under various flow rates. Each $\mathrm{CO}_{2}$ injection lasted for $0.2 \mathrm{~s}$, the time interval between two injections was set to $9.8 \mathrm{~s}$. A component of the gas exiting the tube flowed into a LI-COR $\mathrm{CO}_{2}$ non-dispersive infrared (NDIR) analyzer (model LI-6252). The experiment setup is shown in Fig. 2. A series of experiments were carried out by using $\mathrm{N}_{2}$ gas flow rates of $14,7.7,6.6,5.4$, and $3.9 \mathrm{~L} \mathrm{~min}^{-1}$, which correspond to ram pressures of $110 \mathrm{mbar}, 50 \mathrm{mbar}$, $40 \mathrm{mbar}$, $30 \mathrm{mbar}$, and $20 \mathrm{mbar}$ in front of the WASP tube, respectively. The peak shapes are shown in Fig. 3. The full width at half maximum (FWHM) of the $\mathrm{CO}_{2}$ peaks traversing the entire length of tubing was then compared with direct injection of $\mathrm{CO}_{2}$ peaks into a flow of $\mathrm{N}_{2}$ gas (at the same flow rate) but not going through the WASP tube (Fig. 4). The FWHM of the $\mathrm{CO}_{2}$ peaks that went through the WASP tube (hereafter FWHM-WASP) are significantly different from that of the $\mathrm{CO}_{2}$ peaks directly injected into the $\mathrm{CO}_{2}$ analyzer (hereafter FWHM-direct) under all the flow rate conditions (paired $t$ test, $5 \%$ significance level). The FWHM-direct values (0.84$1.2 \mathrm{~s}$ ) are smaller than the FWHM-WASP values (1.5-4.4 s) and remain relatively constant. The FWHM-WASP with a flow rate of $14 \mathrm{~L} \mathrm{~min}^{-1}(1.5 \mathrm{~s})$ is very close to the FWHMdirect values even though they are statistically different from each other.

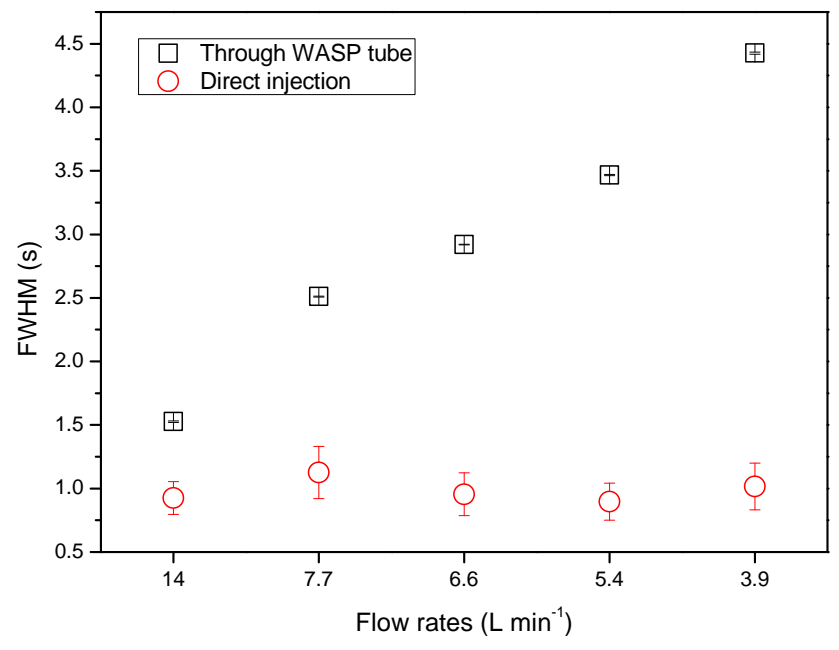

Fig. 4. The full width at half maximum (FWHM) of the $\mathrm{CO}_{2}$ peaks as shown in Fig. 3. Each data point corresponds to the mean FWHM value of the five $\mathrm{CO}_{2}$ peaks under each condition. Error bars indicate one standard deviation.

\subsubsection{Diffusion effects under different storage times}

Once a sample is collected, it must be transported from the airport to the laboratory. During this storage time, molecular diffusion will occur, although that effect is expected to be small, since the transport time is relatively short (typically less than an hour) and concentration gradients for the species of interest should be small. We performed laboratory tests to determine the effect of sample storage. WASP samples injected with pulses of propene were stored for different times, from several minutes up to $14 \mathrm{~h}$, and then analyzed by PTR-TOF-MS (Fig. 5). The FWHM for the propene peaks are $5.1 \pm 0.40 \mathrm{~s}$ (mean \pm 1 standard deviation, $n=6), 4.8 \pm 0.21 \mathrm{~s}, 4.8 \pm 0.38 \mathrm{~s}, 5.0 \pm 0.40 \mathrm{~s}, 5.2 \pm 0.38 \mathrm{~s}$, and $5.7 \pm 0.30 \mathrm{~s}$, respectively, with increasing storage time as shown in Fig. 5. There are no significant differences when comparing the FWHM of $30 \mathrm{~min}$ to $5 \mathrm{~h}$ storage times with $1 \mathrm{~min}$ storage time (paired $t$ test, $5 \%$ significance level). However, for storage time of $14 \mathrm{~h} 35 \mathrm{~min}$, the FWHMs are significantly different from the $1 \mathrm{~min}$ storage time. It is evident that peak smearing resulting from molecular diffusion of propene is small for storage times of less than $5 \mathrm{~h}$. Since analyses typically will be within $1 \mathrm{~h}$ of sample collection, we find this effect to be negligible, especially when considering the diffusion rate is determined primarily by the second derivative of the concentration gradient, and measured concentration gradients in vertical profiles will be many times smaller than the several percent propene gradient used here. Thus we consider the results here as upper limits. In any case, if significant molecular diffusion in a WASP sample did occur, it would be noticeable by the increasing peak width of the pulsed tracer within that sample. The coiled tube was not heated during sample collection, because no significant 


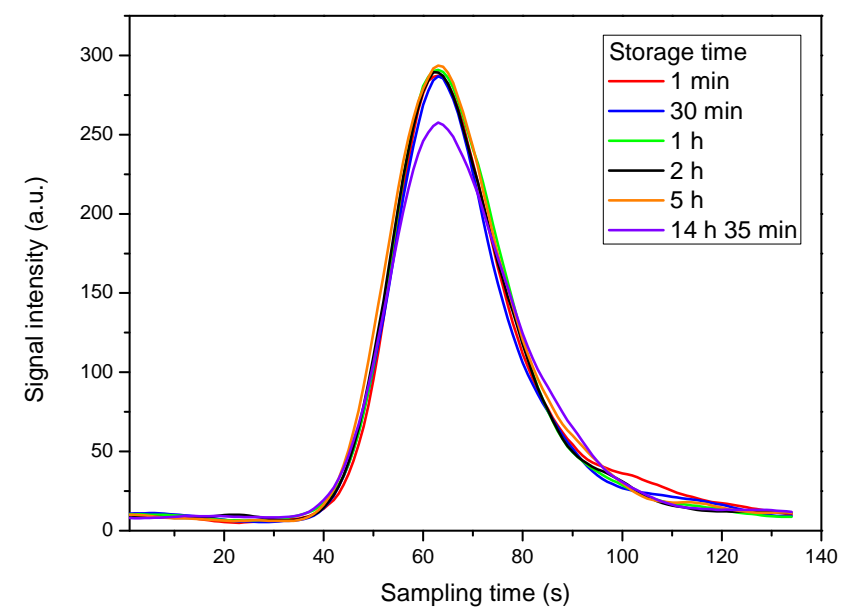

Fig. 5. The intensity of propene signals as a function of sample storage time. Each propene injection lasted for $0.2 \mathrm{~s}$, the time interval between two injections was set to $19.8 \mathrm{~s}$.

differences occurred when running calibration gas mixture through the tube under room temperature. The calibration gas contained chemical species of interest, including isoprene (1 ppmv) and MVK (1 ppmv).

\subsubsection{Peak shape under lower withdrawal flow rate}

After a sample arrives at the laboratory, the front end of the tube is connected to the sample inlet of the PTR-TOF-MS (Sect. 2.3). At the back end of the tubing is a supply of zero air. The PTR-TOF-MS draws in sample air at a set flow rate; in this case an inlet flow rate of $500 \mathrm{sccm}$ is used (this is the maximum). This flow rate corresponds to a velocity of $0.16 \mathrm{~m} \mathrm{~s}^{-1}$ through the WASP tube, which is significantly lower than that during sample collection, and well below the threshold for turbulent flow. The low flow rate during sample extraction is expected to have the largest impact on sample integrity resulting from laminar and Poiseuille flow.

To quantify this effect we filled the tube with propene pulse injections into a pure $\mathrm{N}_{2}$ gas stream at mass flow rate of $3.9 \mathrm{~L} \mathrm{~min}^{-1}$. Propene was pulse injected into the $\mathrm{N}_{2}$ stream entering the tube every $9.8 \mathrm{~s}$, each injection lasted for $0.2 \mathrm{~s}$. After the coiled tube was filled with the propene-spiked $\mathrm{N}_{2}$ gas, the valves on both ends of the WASP are closed. The PTR-TOF-MS inlet was then connected to the back end of the coiled tube with both valves opened and the samples were analyzed under a withdrawal flow rate of $500 \mathrm{sccm}$. We then repeated the above experiment, but this time the PTR-TOFMS inlet was connected to the front end of the coiled tube and the withdrawal flow direction was reversed as compared to the flow direction of the $\mathrm{N}_{2}$ gas stream. The experimental setup is shown in Fig. 2c.

The experiment's results are shown in Fig. 6. The propene signals obtained under different withdrawal flow directions are aligned together along the same coiled tube setup. Since

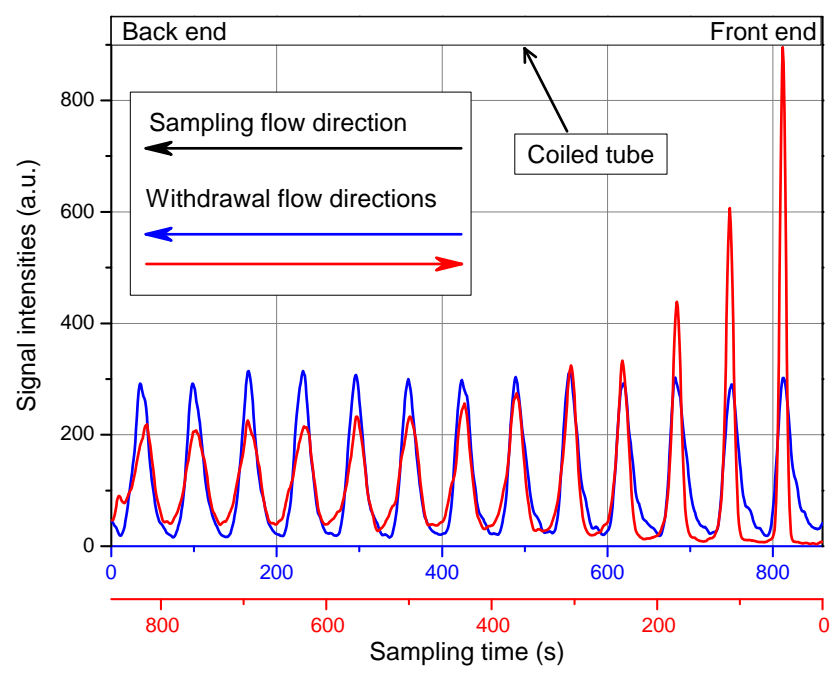

Fig. 6. The intensity of propene signals under different withdrawal flow directions. The blue line indicates that the inlet of PTR-TOFMS is connected to the back end of the WASP tube, the red line indicates that the inlet of PTR-TOF-MS is connected to the front end of the WASP tube. The color of the $x$ axis labels correspond to the withdrawal flow directions. The coiled tube setup is shown on the top of the figure.

the withdrawal flow direction was reversed for the red line, from left to right of the $x$ axis, the last peak is the first peak eluted from the coiled tube, while for the blue line, the first peak is the first peak eluted from the coiled tube. It is evident that when the withdrawal flow direction is the same as the $\mathrm{N}_{2}$ gas flow direction, the peak height and width remain relatively uniform throughout the entire coiled tube. When the withdrawal flow direction was reversed, the peak height decreases as a function of the sampling time while the peak width increases at the same time. The total sampling time of the PTR-TOF-MS during each experiment is less than $20 \mathrm{~min}$, the instrument sensitivity is stable during this time period, thus the different peak shapes under those two experiments are due to the diffusion of propene under different travel distances inside the coiled tube. When the withdrawal flow direction is the same as the $\mathrm{N}_{2}$ gas flow direction, all the propene peaks travel the same distance, i.e., the entire length of the coiled tube. When the withdrawal flow direction is reversed, the first eluted peak traveled the shortest distance inside the coiled tube, while the last eluted peak had to travel almost twice the length of the coiled tube and experience the longest residence time. During the field campaign (discussed in the next section), the withdrawal flow direction was reversed as compared to the WASP sampling direction due to logistic setups. However, in future campaigns, the PTR-TOF-MS withdrawal flow direction should be the same as the WASP sampling flow direction.

Aside from 304 stainless steel tubing, PTFE tubing and silco-treated stainless steel tubing were also tested both in 
the laboratory and during previous field campaigns. For the PTFE tubing, the propene peak centers shifted away from each other after the transportation from the airport to the ground laboratory, i.e., the distance between two adjacent peaks were significantly different from each other. This could be due to the low friction of PTFE material, therefore, for this reason, it was not used during this campaign. For silcotreated stainless steel tubing, wall loss of VOC species (e.g., isoprene, MVK) were generally lower than 304 stainless steel (data not shown here), both materials did not cause uneven distances between two adjacent propene peaks. However, long enough ( $150 \mathrm{~m}$ length) silco-treated stainless steel tubing was not available during the PINOT-NOIR campaign, thus we chose to use the 304 stainless steel tubing since the wall loss of target chemical species (e.g., isoprene, MVK) is still within tolerance, even though slightly more than silcotreated stainless steel tubing.

\subsection{Application of WASP during the PINOT-NOIR campaign}

The LI-COR $\mathrm{CO}_{2}$ NDIR analyzer was used for many of the diagnostic experiments, because of its portability and ease of use. However the WASP was really designed for the quantification of reactive gases. Specifically we are interested in measuring the vertical profiles of gases such as isoprene, MVK, MACR, monoterpenes, acetaldehyde, and other reactive species.

In situ collection of ambient air samples were carried out on 9 and 10 August 2012 during the intensive field campaign Particle Investigations at a Northern Ozarks Tower - $\mathrm{NO}_{\mathrm{x}}$, Oxidants, Isoprene Research (PINOT-NOIR). The sampling site was located at the Baskett Research and Education Area (BREA, $38^{\circ} 44^{\prime} 24^{\prime \prime} \mathrm{N}, 92^{\circ} 12^{\prime} 0^{\prime \prime} \mathrm{W}$ ), which is an oak-dominated forest managed by the University of Missouri (http://aes.missouri.edu/baskett/). The study involved measurements using a high resolution time of flight mass spectrometer (HTOF-MS, Tofwerks, Switzerland). de Gouw and Warneke (2007) reviewed the operational mode for $\mathrm{H}_{3} \mathrm{O}^{+}$ chemistry using the PTR-MS technique in detail. Briefly, measurements were performed using a PTR-TOF-MS 8000 apparatus from Ionicon Analytik GmbH, Innsbruck (Austria) (Jordan et al., 2009), where the ionization conditions in the drift tube were controlled by drift voltage $(530 \mathrm{~V})$, drift temperature $\left(60^{\circ} \mathrm{C}\right)$ and drift pressure $(2.3 \mathrm{mbar})$ resulting in an $E / N$ of about $115 \mathrm{Td}$. A capillary inlet heated to $60^{\circ} \mathrm{C}$ was used as a transfer line, which was flushed at a flow rate of $250 \mathrm{sccm}$. The transfer line was connected to an unheated $1 / 8$ in. OD Teflon ${ }^{\circledR}$ line $(\sim 5 \mathrm{~m}$ length $)$, which was connected to the $150 \mathrm{~m}$ WASP coiled tube. The signal on $\mathrm{m} / z=43^{+}$ was used to monitor propene pulses that allowed correlation with the GPS altitude signal and was used to reconstruct the air mass history as a function of position in the coiled tube. A custom built calibration system was used to dilute a gravimetrically prepared VOC standard that included

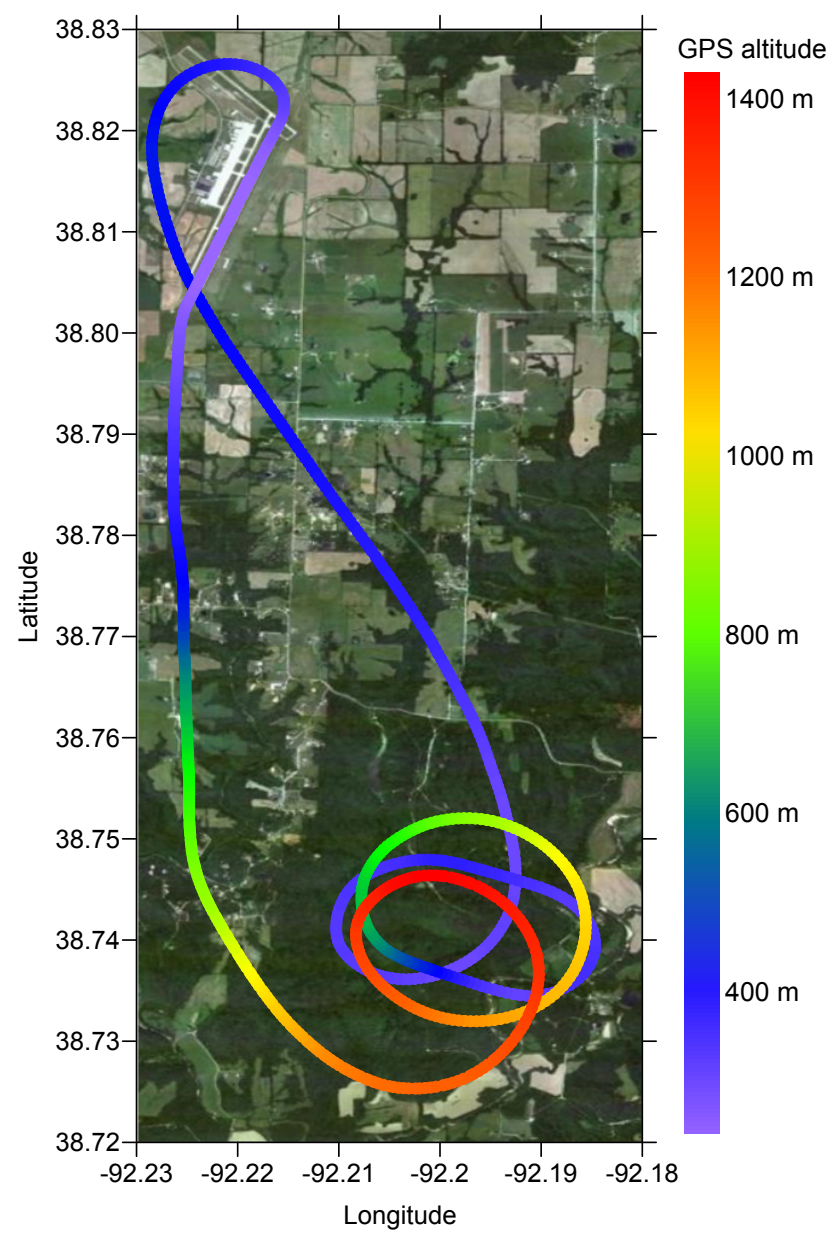

Fig. 7. The flight track of flight 5 conducted on 10 August 2012. The satellite map is obtained from Google Earth.

isoprene. The gas standard was gravimetrically prepared in house and contained isoprene ( $7.25 \mathrm{ppmv})$ and camphene (4.87 ppmv). The dynamically diluted standard gas was directly sampled by the PTR-TOF-MS inlet, the WASP system was not included as part of the calibration experiment. The limits of detection ( $3 \sigma$ uncertainty of zero air background measurement) for isoprene and camphene were calculated to be $<0.055 \mathrm{ppbv}$ and $<0.020 \mathrm{ppbv}$, respectively (1 min integration time). The total uncertainty of the sensitivity calibration was estimated to be within $\pm 15 \%$.

A representative flight track is shown in Fig. 7. The aircraft took off at the Columbia Regional Airport $(\sim 10 \mathrm{~km}$ to the north of the BREA). For each flight the air sampling usually started at $\sim 30 \mathrm{~m}$ above the canopy top, climbing to a maximum altitude of $1000-1200 \mathrm{~m}$ above the ground. The time interval between two tracer gas injections was set to $9.6 \mathrm{~s}$ and each injection lasted for $0.2 \mathrm{~s}$. A total of 13 tracer gas peaks were injected into the air stream and the total collection time was $\sim 2 \mathrm{~min}$. After this point the WASP was switched back to inactive mode and the aircraft started to descend and return 


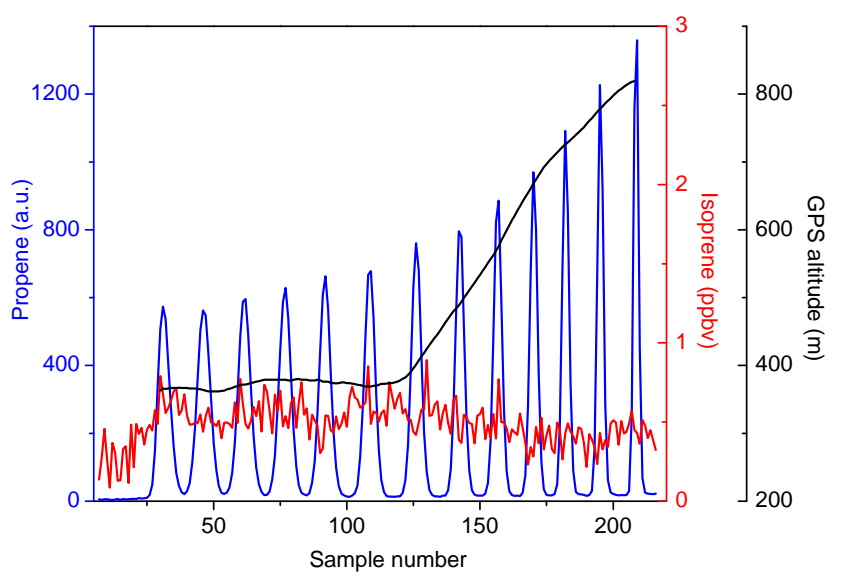

Fig. 8. The concurrent mixing ratio of isoprene with the count rate of propene. The GPS altitude is interpolated based on the peak position of propene. Data shown here are for flight 4 during the PINOTNOIR campaign. See text for detailed explanation.

to the airport. The total time for each flight was $\sim 13 \mathrm{~min}$. After each flight, the coiled tube was transported from the airport to the BREA flux tower field laboratory where the samples were analyzed by the PTR-TOF-MS. The time for the transportation between the airport and the field laboratory was $\sim 40 \mathrm{~min}$. The time interval between two flights was $\sim 2.5 \mathrm{~h}$. A total of 8 flights were conducted, which retrieved 8 profiles: three on 9 August and five on 10 August. Here we discuss the vertical profiles obtained on 10 August (flights 4-8).

\section{Results and discussion}

\subsection{Reconstructing altitude profiles for WASP samples}

During sampling, the altitude information of the ambient air is preserved by pulsing timed tracer gas peaks into the air sample stream right before it enters the coiled tube. The GPS time, altitude, and meteorological data are recorded together by the AIMMS-20 system. After each flight, the air sample inside the coiled tube is measured by using the PTR-TOFMS and the counts rate of the tracer gas as well as the VOC species are recorded simultaneously. Figure 8 shows the profile of tracer gas (propene) and mixing ratios of isoprene from flight 4 of the PINOT-NOIR campaign. To obtain the GPS altitude for each VOC mixing ratio data point in Fig. 8, the sampling time of the first injected tracer gas peak (the first peak shown in Fig. 8) is synchronized with the corresponding GPS time from the AIMMS-20 system. Then the sampling time of all the tracer peaks are calculated according to the known time sequence. The sampling time for all the VOC mixing ratio data points between two tracer gas peaks are calculated by interpolation. The GPS altitudes of all the VOC mixing ratio data points are then calculated by inter-

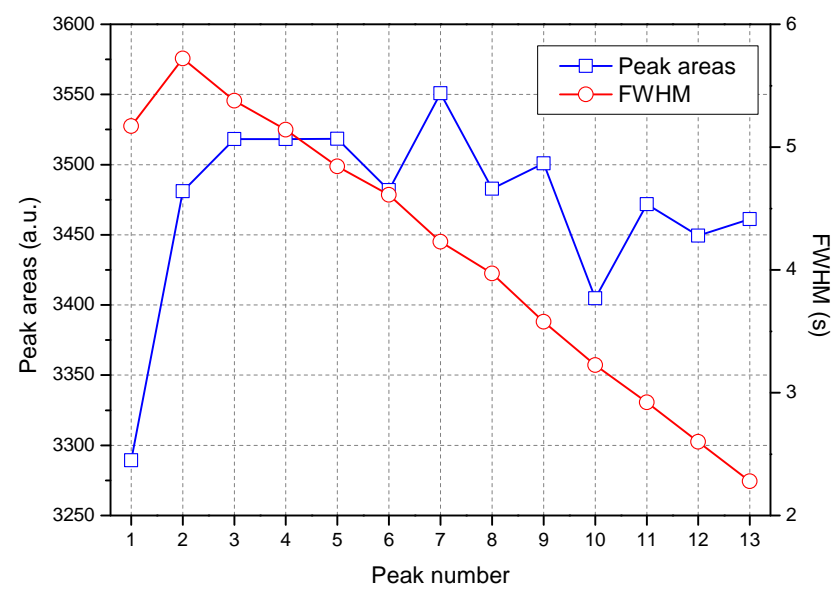

Fig. 9. The integrated peak areas of the propene peaks as shown in Fig. 8. Also shown is the FWHM of all the corresponding peaks.

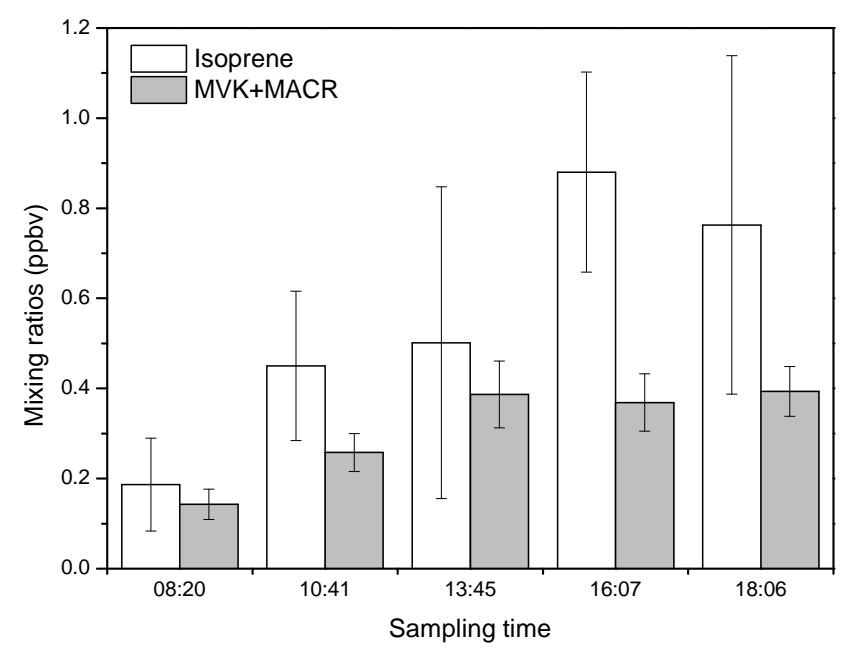

Fig. 10. The mean mixing ratios of isoprene and MVK + MACR during flights $4-8$ conducted on 10 August 2012. The error bars indicate 1 standard deviation for each flight.

polating the calculated sampling time on the GPS time and a typical output is shown in Fig. 8. The meteorological data are obtained at the same time.

The smearing of the propene peaks is significant especially for the earlier injected peaks at lower sampling altitude. This effect is demonstrated in Sect. 2.2.3 by pulling propene-spiked $\mathrm{N}_{2}$ gas at the same withdrawal flow rate as the field campaign. To quantify this effect during the field sampling and measurement, the areas and FWHM are calculated for all the propene peaks (Fig. 9). Except for the first injected peak, the FWHM keep decreasing as the peak height increases. The peak areas are not significantly different from the mean value (one sample $t$ test, $5 \%$ significance level). The deviation of the first injected peak may be due to the disturbance from ambient room air during measurement. 

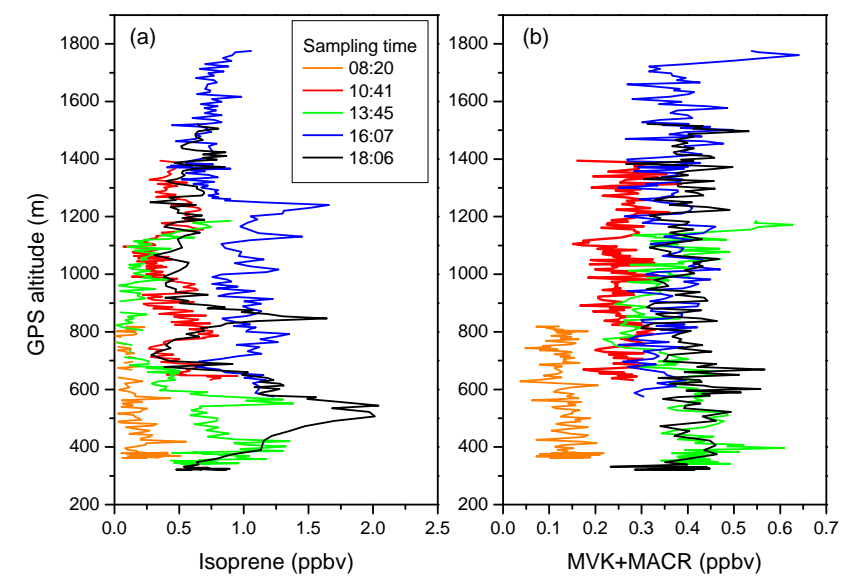

Fig. 11. The vertical profiles of (a) isoprene and (b) MVK + MACR on 10 August 2012. The sampling times indicate the beginning of each flight in local time. Altitude is given in meters above mean sea level; elevation of Columbia is $200 \mathrm{~m}$ MSL.

\subsection{VOC mixing ratios during PINOT-NOIR}

Isoprene $\left(\mathrm{C}_{5} \mathrm{H}_{8}\right)$ is the dominant BVOC species emitted from an oak-dominated forest. The mean mixing ratios of isoprene and its major photooxidation products, MVK + MACR, for flights $4-8$ are shown in Fig. 10. The mean mixing ratio of isoprene is lowest in the early morning and builds up to reach a maximum value of $0.88 \pm 0.22$ (mean \pm 1 standard deviation) ppbv at 16:07 in the afternoon. After this point the mixing ratio of isoprene drops slightly to $0.76 \pm 0.38 \mathrm{ppbv}$ at 18:06 CST. Figure 10 shows that the photooxidation products, MVK + MACR, also have a low mean mixing ratio in the early morning. However, the mean mixing ratio of MVK + MACR remained relatively stable throughout the afternoon. The variability of the isoprene mixing ratio is relatively large especially for the three flights carried out in the afternoon when the isoprene mean mixing ratios are higher. In contrast, the variability of MVK + MACR is relatively low and remains relatively consistent among all the flights.

The vertical profiles of isoprene (Fig. 11) reveal some interesting features of its distribution throughout the air column from above the forest canopy up to $\sim 1800 \mathrm{~m}$ (mean sea level, MSL; the site elevation is about $200 \mathrm{~m}$ ). At the beginning of the day the mixing ratios of isoprene are low, in some cases below the detection limit of the PTR-TOF-MS, and there is no obvious vertical variation. In the early afternoon at $13: 45$, the isoprene mixing ratios increased to $1.0-1.5 \mathrm{ppbv}$ between the altitudes of 300 and $600 \mathrm{~m}$. The mixing ratios drop significantly above $600 \mathrm{~m}$ and increase slightly at the top of this flight $(\sim 1200 \mathrm{~m})$. For the flight taken at 16:07, the isoprene mixing ratio remains relatively stable at $\sim 1.0 \mathrm{ppbv}$ at altitudes of $600-1200 \mathrm{~m}$ and then drops sharply to 0.50 $0.70 \mathrm{ppbv}$ above $1300 \mathrm{~m}$. The vertical profile of isoprene from the last flight shows two distinct peaks at $\sim 550$ and $\sim$ $850 \mathrm{~m}$, respectively. The vertical profiles of MVK + MACR
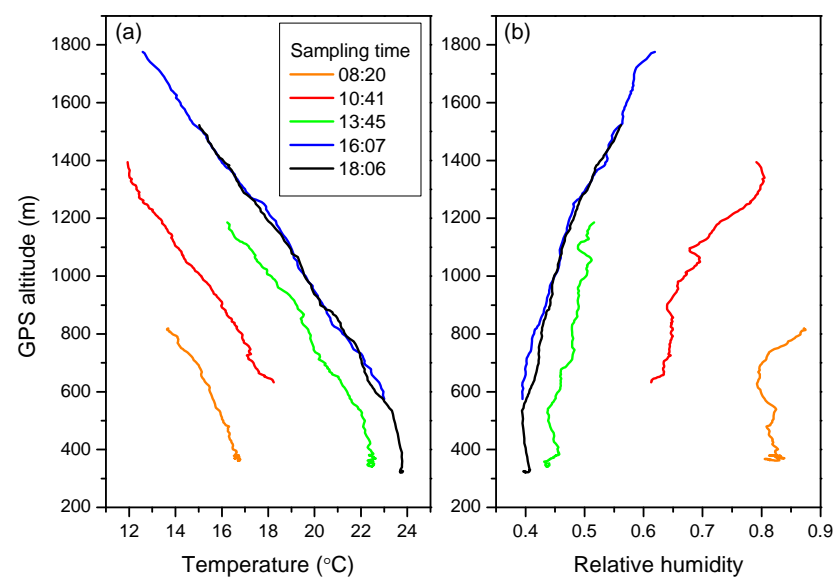

Fig. 12. The vertical profiles of (a) ambient air temperature and (b) relative humidity on 10 August 2012. The sampling time indicates the beginning of each flight in local time. Altitude is given in meters above mean sea level; elevation of Columbia is $200 \mathrm{~m}$ MSL.

do not show significant variations throughout the air column. The largest variation occurs at early afternoon.

The vertical profiles of isoprene and MVK + MACR are affected by factors including surface emission and deposition, vertical turbulent transportation within the boundary layer, entrainment from the free troposphere and chemical losses. The biogenic emission of isoprene is light-dependent, resulting in low mixing ratios in the early morning immediately after sunrise. During the rest of the day, the isoprene mixing ratios build up. The increasing isoprene emission source is somewhat balanced by the mixing ratio decrease due to higher vertical transport and chemical losses. Another factor that can contribute to the variation of the vertical profiles of isoprene is transportation from other regions. At a sampling height of $\sim 1000 \mathrm{~m}$, the footprint size is on the order of $10^{4} \mathrm{~m}$, which means the isoprene sampled over the BREA area could be transported from other areas with either higher or lower isoprene emissions. On the other hand, the mixing ratios of MVK + MACR are controlled mainly by in situ photooxidation of isoprene, thus the vertical structures are relatively uniform throughout the vertical column.

\subsection{Footprint of the emission sources}

The airborne sampling and subsequent analysis can determine the VOC's mixing ratios above the forest canopy to a higher altitude than any ground-based tower measurement. Meanwhile, it is necessary to estimate the upwind "source areas" that contribute to those measured VOC concentrations or fluxes at a higher altitude. The footprint size and location is dependent on the measurement height, the atmospheric stability conditions within the planetary boundary layer (PBL), and the roughness of the surface (Kljun et al., 2004; Horst, 1999; Baldocchi, 1997; Kaharabata et al., 1999). For this campaign, the top sampling altitude 
is $\sim 1000 \mathrm{~m}$, much higher than tower measurements, which are typically $<100 \mathrm{~m}$ and are at most $\sim 300 \mathrm{~m}$. By using a footprint function facilitated with a scaling approach (Kljun et al., 2004), we are able to obtain the estimated footprint size for the aircraft-based measurements. As indicated by the vertical temperature and relative humidity profiles (Fig. 12), the PBL height during the sampling period on 10 August was $>1500 \mathrm{~m}$. The estimated peak values of the footprint varied by two orders of magnitude $\left(10^{2}-10^{4} \mathrm{~m}\right.$, data not shown), depending on different sampling heights.

A more comprehensive analysis of the vertical profiles and the associated fluxes can be accomplished using the horizontal wind speeds and turbulence at different altitudes, which are available from the on-board AIMMS-20 system, as well as other constraints.

\section{Conclusions}

The WASP system successfully collected highly resolved vertical profiles of air from near the surface to a height of $1000-1500 \mathrm{~m}$. The aircraft sampling conditions used for this collection ensures that the results are not affected significantly by molecular diffusion or laminar flow characteristics. A unique component of the WASP is the pulse injection tracer system that allows us to chemically timestamp the air sample throughout the tube length. Using the GPS data from the AIMMS-20 system, we can reconstruct the position of the sampled air to within a few meters' altitude (this is dependent upon the type of post-collection analysis; in our case the sample frequency of the PTR-TOF-MS allows for high resolution vertical positioning). The pulse injection system also allows us to confirm the degree of smearing that may take place during air sample handling. We find that extraction of the air sample post-collection has the greatest impact on the "smearing" of the collected profiles; however the effect is small, and does not take away from the usefulness of such observations. The WASP fills a gap that extends above towerbased vertical profiling systems that are typically limited to the lowest $100 \mathrm{~m}$ and at most $300 \mathrm{~m}$. Tethered balloon sampling systems can probe higher but do not provide the vertical resolution of the WASP or the capability for easy movement to different locations. The WASP offers many advantages over these alternatives and will be deployed in future campaigns that focus on trace gas emissions.

Acknowledgements. The authors thank T. Evans, B. Piscitello, and K. Lamont for initial construction and testing of the WASP system. R. Seco is thanked for assisting the measurement of air samples during the field campaign. We are grateful for discussions and logistical support from D. Lenschow and D. Knopf. This work was supported in part by the National Science Foundation (EAGER grant \# AGS-1133418 and AGS-1098464). The aircraft was provided and operated by UltraPure Air, LLC. T. Karl was also supported by the EC Seventh Framework Program (Marie Curie
Reintegration Program, “ALP-AIR”, grant \# 334084). The National Center for Atmospheric Research is operated by the University Corporation for Atmospheric Research under sponsorship from the National Science Foundation.

Edited by: D. Heard

\section{References}

Atkinson, R. and Arey, J.: Gas-phase tropospheric chemistry of biogenic volatile organic compounds: a review, Atmos. Environ., 37 S197-S219, doi:10.1016/S1352-2310(03)00391-1, 2003.

Baldocchi, D.: Flux footprints within and over forest canopies, Bound.-Lay. Meteorol., 85, 273-292, doi:10.1023/A:1000472717236, 1997.

Beswick, K. M., Gallagher, M. W., Webb, A. R., Norton, E. G., and Perry, F.: Application of the Aventech AIMMS20AQ airborne probe for turbulence measurements during the Convective Storm Initiation Project, Atmos. Chem. Phys., 8, 5449-5463, doi:10.5194/acp-8-5449-2008, 2008.

Bouvier-Brown, N. C., Goldstein, A. H., Gilman, J. B., Kuster, W. C., and de Gouw, J. A.: In-situ ambient quantification of monoterpenes, sesquiterpenes, and related oxygenated compounds during BEARPEX 2007: implications for gas- and particle-phase chemistry, Atmos. Chem. Phys., 9, 5505-5518, doi:10.5194/acp-9-5505-2009, 2009.

de Gouw, J. and Warneke, C.: Measurements of volatile organic compounds in the earths atmosphere using proton-transferreaction mass spectrometry, Mass Spectrom. Rev., 26, 223-257, doi:10.1002/mas.20119, 2007.

DiGangi, J. P., Boyle, E. S., Karl, T., Harley, P., Turnipseed, A., Kim, S., Cantrell, C., Maudlin, R. L., Zheng, W., Flocke, F., Hall, S. R., Ullmann, K., Nakashima, Y., Paul, J. B., Wolfe, G. M., Desai, A. R., Kajii, Y., Guenther, A., and Keutsch, F. N.: First direct measurements of formaldehyde flux via eddy covariance: implications for missing in-canopy formaldehyde sources, Atmos. Chem. Phys., 11, 10565-10578, doi:10.5194/acp-1110565-2011, 2011.

Fuentes, J. D., Lerdau, M., Atkinson, R., Baldocchi, D., Bottenheim, J. W., Ciccioli, P., Lamb, B., Geron, C., Gu, L., Guenther, A., Sharkey, T. D., and Stockwell, W.: Biogenic hydrocarbons in the atmospheric boundary layer: A review, B. Am. Meteorol. Soc., 81, 1537-1575, 2000.

Greenberg, J. P., Guenther, A., Zimmerman, P., Baugh, W., Geron, C., Davis, K., Helmig, D., and Klinger, L. F.: Tethered balloon measurements of biogenic VOCs in the atmospheric boundary layer, Atmos. Environ., 33, 855-867, doi:10.1016/S13522310(98)00302-1, 1999.

Guenther, A., Geron, C., Pierce, T., Lamb, B., Harley, P., and Fall, R.: Natural emissions of non-methane volatile organic compounds; carbon monoxide, and oxides of nitrogen from North America, Atmos. Environ., 34, 2205-2230, doi:10.1016/S13522310(99)00465-3, 2000.

Horst, T. W.: The footprint for estimation of atmosphere-surface exchange fluxes by profile techniques, Bound.-Lay. Meteorol., 90, 171-188, doi:10.1023/A:1001774726067, 1999. 
Jordan, A., Haidacher, S., Hanel, G., Hartungen, E., Mark, L., Seehauser, H., Schottkowsky, R., Sulzer, P., and Mark, T. D.: A high resolution and high sensitivity proton-transfer-reaction time-offlight mass spectrometer (PTR-TOF-MS), Int. J. Mass Spectrom., 286, 122-128, doi:10.1016/j.ijms.2009.07.005, 2009.

Kaharabata, S. K., Schuepp, P. H., and Fuentes, J. D.: Source footprint considerations in the determination of volatile organic compound fluxes from forest canopies, J. Appl. Meteorol., 38, 878884, 1999.

Karl, T., Guenther, A., Yokelson, R. J., Greenberg, J., Potosnak, M., Blake, D. R., and Artaxo, P.: The tropical forest and fire emissions experiment: Emission, chemistry, and transport of biogenic volatile organic compounds in the lower atmosphere over Amazonia, J. Geophys. Res.-Atmos., 112, D18302, doi:10.1029/2007JD008539, 2007.

Karl, T., Misztal, P. K., Jonsson, H. H., Shertz, S., Goldstein, A. H., and Guenther, A. B.: Airborne flux measurements of BVOCs above Californian oak forests: Experimental investigation of surface and entrainment fluxes, $\mathrm{OH}$ densities and Dahmköhler numbers, J. Atmos. Sci., doi:10.1175/JAS-D-13-054.1, 2013.

Kesselmeier, J. and Staudt, M.: Biogenic volatile organic compounds (VOC): An overview on emission, physiology and ecology, J. Atmos. Chem., 33, 23-88, doi:10.1023/A:1006127516791, 1999.

Kim, S., Karl, T., Guenther, A., Tyndall, G., Orlando, J., Harley, P., Rasmussen, R., and Apel, E.: Emissions and ambient distributions of Biogenic Volatile Organic Compounds (BVOC) in a ponderosa pine ecosystem: interpretation of PTR-MS mass spectra, Atmos. Chem. Phys., 10, 1759-1771, doi:10.5194/acp-101759-2010, 2010.
Kljun, N., Calanca, P., Rotachhi, M. W., and Schmid, H. P.: A simple parameterisation for flux footprint predictions, Bound.-Lay. Meteorol., 112, 503-523, doi:10.1023/B:BOUN.0000030653.71031.96, 2004.

Laothawornkitkul, J., Taylor, J. E., Paul, N. D., and Hewitt, C. N.: Biogenic volatile organic compounds in the Earth system, New Phytol., 183, 27-51, doi:10.1111/j.1469-8137.2009.02859.x, 2009.

Müller, M., Graus, M., Ruuskanen, T. M., Schnitzhofer, R., Bamberger, I., Kaser, L., Titzmann, T., Hortnagl, L., Wohlfahrt, G., Karl, T., and Hansel, A.: First eddy covariance flux measurements by PTR-TOF, Atmos. Meas. Tech., 3, 387-395, doi:10.5194/amt-3-387-2010, 2010.

Warneke, C., de Gouw, J. A., Del Negro, L., Brioude, J., McKeen, S., Stark, H., Kuster, W. C., Goldan, P. D., Trainer, M., Fehsenfeld, F. C., Wiedinmyer, C., Guenther, A. B., Hansel, A., Wisthaler, A., Atlas, E., Holloway, J. S., Ryerson, T. B., Peischl, J., Huey, L. G., and Hanks, A. T. C.: Biogenic emission measurement and inventories determination of biogenic emissions in the eastern United States and Texas and comparison with biogenic emission inventories, J. Geophys. Res.-Atmos., 115, D00F18, doi:10.1029/2009JD012445, 2010. 